\title{
Lane Keeping Maneuvers Using Proportional Integral Derivative (PID) and Model Predictive Control (MPC)
}

\author{
Moveh Samuel ${ }^{1}$, Maziah Mohamad ${ }^{2}$, Mohamed Hussein ${ }^{3}$, Shaharil Mad Saad ${ }^{4}$ \\ 1, 2,3,4 Department of Applied Mechanics and Design, School of Mechanical Engineering, Universiti Teknologi \\ Malaysia, Malaysia 81310 UTM Skudai Johor Malaysia \\ Email: *1 smoveh@yahoo.com, ${ }^{2}$ maziah@utm.my, ${ }^{3}$ mohamed@mail.fkm.utm.my, ${ }^{4}$ shaharil@utm.my \\ *Corresponding Author
}

\begin{abstract}
Safety has been a concern as a result of the high rate of road accidents and has led to the development of driver assistance systems which has become a very active research area. This study presents the performance and robustness analysis of a model predictive control (MPC) and proportional integral derivative control (PD) for lane-keeping manoeuvres of a self driving car using image processing. Simulation studies were carried using a vehicle model known as single-tracked bicycle model developed in MATLAB/SIMULINK environment as well as a vision dynamic system. Both PD and MPC were simulated to follow a particular desired reference trajectory by controlling the steering angle. Performance and robustness analysis were carried out and the simulation results showed that the proposed control system for the PD control achieved its objective even though it was less robust in maintaining its performance under various conditions like vehicle load change, different longitudinal speed and different cornering stiffness. While in the case of MPC the optimizer made sure that the predicted future trajectory of the vehicle output tracks the desired reference trajectory and was more robust in maintaining its performance under the same conditions as in PD.
\end{abstract}

Keywords - performance, robustness, analysis, lane keeping, manoeuvres autonomous, vehicles

\section{INTRODUCTION}

At the current rate of development, civilization specifically in the Automotive industry and sector like development of auto-electronics technology. Active safety is one of the most studied sector. This has lead to a lot of research in recentent times.

In a study of [1], Safety has become an important issue due to the increasing number of vehicles and road users, resulting in the high rate of road accidents recorded every year. The same study also suggested that the vast majority of these accidents are caused iby driving errors, which can be avoided.

Car manufacturers are now focusing on developing new features to assist drivers, to improve safety and provide comfort. As stated in [2] Advance Driver Assistance System (ADAS) was developed to ensure safety and assistance to a driver with the help of sensors technology and control algorithm, the system help to expand safety, adjust to encompassing and control the vehicle. The system has made considerable progress in a moderately brief period, by the norms of the automobile industries. The innovation apparently has its foundations in highlights that started appearing over 10 years ago, such as adaptive cruise control, or maybe considerably further back to the presentation of driver warning systems like emergency brake lights. According to [3] Some of the most common features that are in cooperated into the ADAS system includes: Collision avoidance system, Adaptive cruise control, automate ibraking, automate lighting, parking assist system, lane departure warning system and a lane-keeping system. This study is focused more on one of such ADAS safety feature lane keeping.

The task of autonomously driving a car involves the following: keeping safe distance vehicles, speed control, lane keeping and lane detection, lane change manoeuvres for overtaking vehicles, obstacle avoidance, establishing the shortest route to a destination, and parking within urban environments. It is a challenging task that has attracted the interest of researchers and research institutions globally. It is believed that autonomously driven vehicles help vehicles in keeping a short but reliable safe distance of $10 \mathrm{~m}-20 \mathrm{~m}$ thereby increasing roads and highways capacity [4]. It helps to provide a reduction in commuting time and autonomous driving leads to optimal use of infrastructures. Remarkable fuel-saving expenses are achieved by automatically controlling vehicles velocity as well as traffic decongestion.

In the automated driving mode of the autonomous car, the vehicle's lateral movement is controlled by a lateral control algorithm. The objective of this algorithm is to keep the vehicle in the centre of its lane. Position and orientation of the vehicle can be determined by the use of sensors such as camera used in identifying the white middle marker and left and right boundaries on the road and also by the use of sensors like Lidar for path tracking techniques both by explicitly or implicitly means. Regardless of the apparent simplicity of discovering white lane marks on the road, it can be extremely hard to find white lane marks on a different kind of road. These problems could be attributed to either of the following; shadows, occlusion by other vehicles, changes in the road surface itself, and different types of lane markings. A good lane detection algorithm 
should be capable of detecting all kind of lane marks from roadways and filter them to estimate an accurate estimate of the vehicle position relative to the lane.

\section{CONTROL DYNAMICS}

The vehicles lateral dynamic has been talked about in [3], where both kinematic and the dynamic models of vehicle lateral motion have been considered. Many control plans have been set up to control the lateral dynamics. [4] and [5] have suggested control laws dependent on Proportional-Integral-Derivative (PID) control, while [6] developed a fuzzy lateral controller. Other control techniques have additionally been utilized, for example, $\mathrm{H} \infty$ control [7], [8], Linear Quadratic Regulator (LQR) [9] and sliding mode control [10], [11], [12]. Additionally, [13] introduced a feedback controller that control lateral dynamics to control back wheel-drive vehicles subject to state limitations. Be that as it may, these control strategies are created dependent on the linearized model of the lateral dynamic, while this paper deals with the control issue for the nonlinear lateral dynamics. Model Predictive Control (MPC) is another technique utilized in the tracking of selfdriving vehicles as seen in [14], [15], [16]. The calculation period of nonlinear MPC is the fundamental impediment of such a methodology: this paper proposes an explanatory answer for the lateral control issue which doesn't cause any calculation loads. [17], [18] have examined the lateral control of autonomously activated four-wheeled vehicles. Comparison of various lateral regulators can be found in [19], [20].

\section{Methodology PREPARE}

The philosophy of the simulation work is always to use simple models, to understand more aspects possible about the physical system. A mathematical model was developed to predict the vehicle desired motion, stability and handling. The major responsibility of the vehicle can be explained based on a linear vehicle model. The vehicle model used in this work was the bicycle model, using an augmented version of two-degree-of-freedom (2DOF) elementary automobile as seen in Fig 1 below. This model was linearized for simplicity and has consistently been proven in various studies [21], [22], [23], [24]. The nomenclatures used are presented in Table 1.

The lane-keeping control was simulated for both PD control and MPC control in Matlab/Simulink environment under various conditions using the parameters in Table 1. Two different analyses were performed: performance and robustness analysis as in [25], [26].

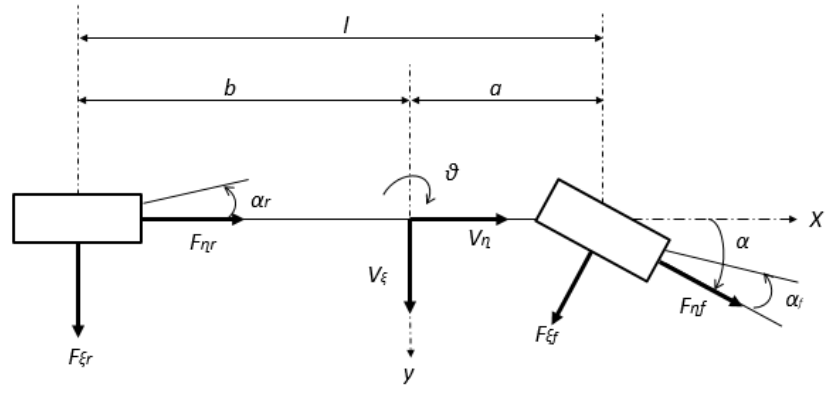

Fig 1. Single-track bicycle model

$$
u(t)=K_{P} e(t)+K_{i} \int e(t) d t+K_{d} \frac{d e}{d t}
$$

Table 1. List of parameters for vehicle dynamic

\begin{tabular}{|c|c|c|}
\hline No & Parameter & Symbol \\
\hline 1 & front tire cornering stiffness & $\mathrm{C}_{\mathrm{af}}$ \\
\hline 2 & rear tire cornering stiffness & $\mathrm{C}_{\mathrm{ar}}$ \\
\hline 3 & vehicle longitudinal velocity & $\mathrm{u}$ \\
\hline 4 & front tire distance & $\mathrm{a}$ \\
\hline 5 & rear tire distance & $\mathrm{b}$ \\
\hline 6 & motor inertia & $\mathrm{I}_{\mathrm{z}}$ \\
\hline 7 & mass of the vehicle & $\mathrm{m}$ \\
\hline 8 & front and rear side slip angle & $\alpha_{\mathrm{f}}, \alpha_{\mathrm{r}}$ \\
\hline 9 & $\begin{array}{c}\text { lateral tire force on front and rear } \\
\text { tires }\end{array}$ & $F_{y f}, F_{y r}$ \\
\hline 10 & $\begin{array}{c}\text { longitudinal/lateral velocity of } \\
\text { vehicle in body reference frame }\end{array}$ & $V$ \\
\hline 11 & yaw rate & $\psi$ \\
\hline 12 & side slipr & \\
\hline
\end{tabular}

\section{RESULTS AND DISCUSSION}

\section{A. Performance and Robustness Analysis}

Performance analysis was being done by simulating the closed-loop system with longitudinal velocities of $\mathrm{Vx}=10$, $15,20,25 \mathrm{~m} / \mathrm{s}$ at a particular look-ahead distance L for both PD and MPC control. Robustness analysis for the PD and MPC closed-loop system was also done at different mass and cornering stiffness of the vehicle.

These simulations were carried out to test the performance and robustness of theses controller under various vehicle load, where the vehicle mass $m$ was varied by increasing the load and the cornering stiffness of the front wheel was varied to see the robustness of the controls under different road conditions as seen [27]. All simulation results are shown below for both controls.

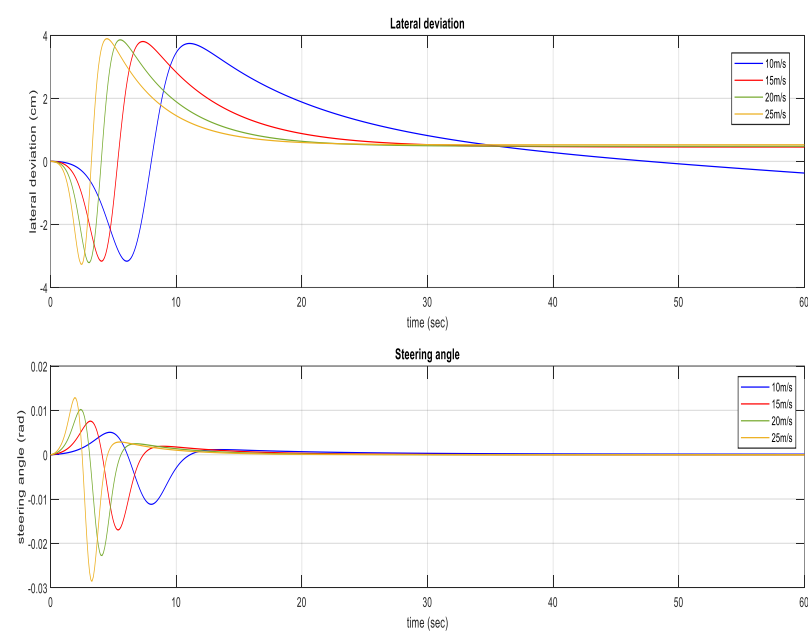

Fig 2. PD performance at different longitudinal velocity $\mathrm{Vx}$

From Fig 2, it was found that the controller was able to compensate for the cross-track error of the system by bringing it to zero as can be seen in the steering angle response. Although the response of the vehicle tends to overshoot at the beginning. This may cause passengers to be 
uncomfortable in a real scenario, before reducing the crosstrack error to zero. Also, it can be observed that changing the longitudinal speed alters the performance. In this case the higher the speed the more the oscillations, but when the vehicle moves at a lower speed the oscillations and overshoot were less. As a conclusion, at a fixed look-ahead distance L, the speed should be varied from high too low for better performance even on a straight and curved road. This is synonymous with the real situation while driving, especially at curved roads, where the speed is lowered for better cornering performance.
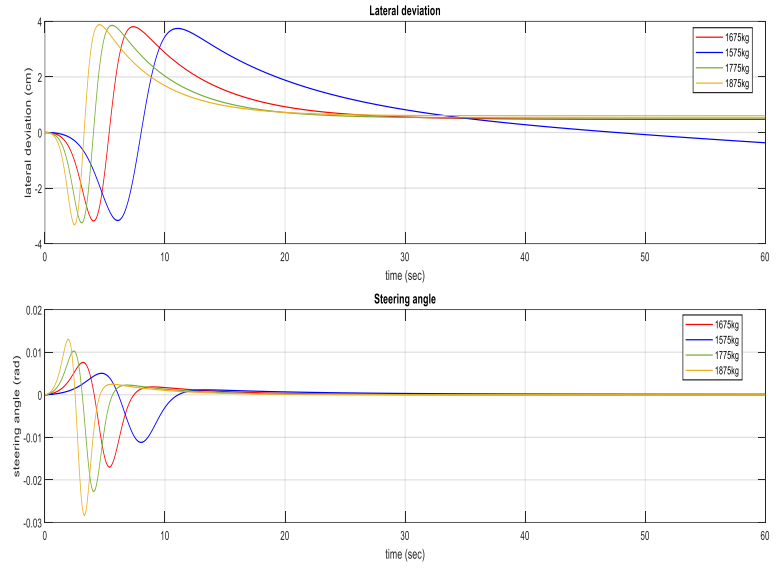

Fig 3. PD Robustness Analysis at different load (mass) Change

The first analysis was done for different values of the vehicle mass as can be seen in Fig 3. This analysis was necessary to check how robust the vehicle will be under various vehicle load, as in the actual case of a real car with more than one passenger in it. This was achieved by simulating at different values of the vehicle mass $m$. Also by changing the load capacity of the vehicle simulation results show that this affected the performance of the control. At low load capacity, the vehicle was more stable, as it can be seen that the controller tries to steer the vehicle to its desire reference trajectory. But when the load capacity was increased, the response changed as can be seen in the figure. At the highest load capacity, the vehicle oscillation increased significantly as the controller tries to steer the vehicle to its desired reference trajectory

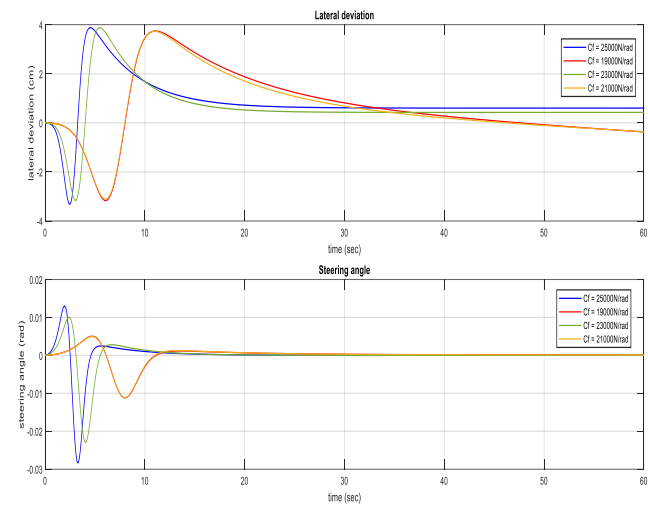

Fig 4. PD Robustness Analysis at different values of front cornering stiffness $\mathrm{C}_{\mathrm{f}}$
Also as seen in Fig 4 the simulation of different value of front cornering stiffness was carried out as in the case of driving the car in different road conditions. In the same manner and pattern, the controller's performance was altered when the front cornering stiffness was altered. When the simulation was carried out at a lower front cornering stiffness, the vehicle was able to follow the desired reference trajectory at a much quicker time as can be seen in figure 4. But when the front cornering stiffness was increased, the vehicle behaviour changed. It can be seen that the vehicle had a wider oscillation before the controller was able to drive the vehicle to follow the desire reference trajectory as can be seen in the figure.

MPC is a feedback control algorithm that uses a model of the vehicle to make predictions about future outputs of the vehicle trajectory. Hence, this section presents an explanation of how the model predictive controller was used for the simulation of the mobile robot.

$$
J=\sum_{i=1}^{p} w_{e} e_{k}+i^{2}+\sum_{i=0}^{p-1} w_{\Delta u} \Delta u_{k}+i^{2}
$$
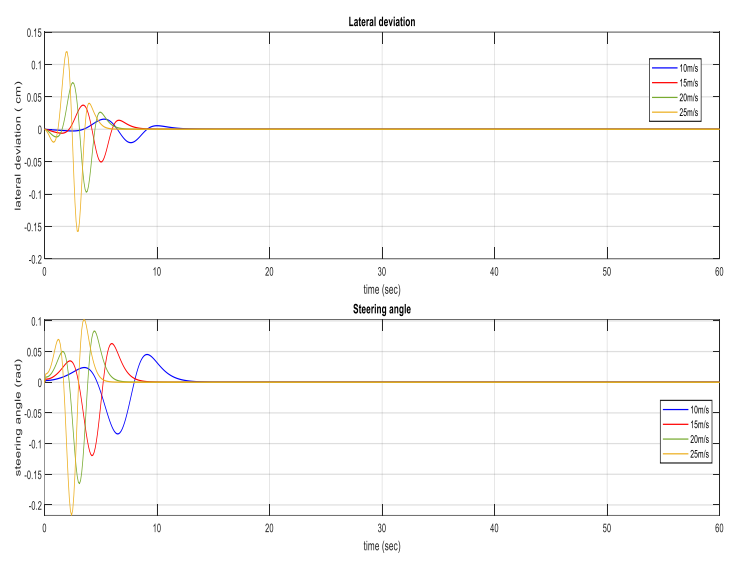

Fig 5. MPC performance at different longitudinal velocity $\mathrm{Vx}$

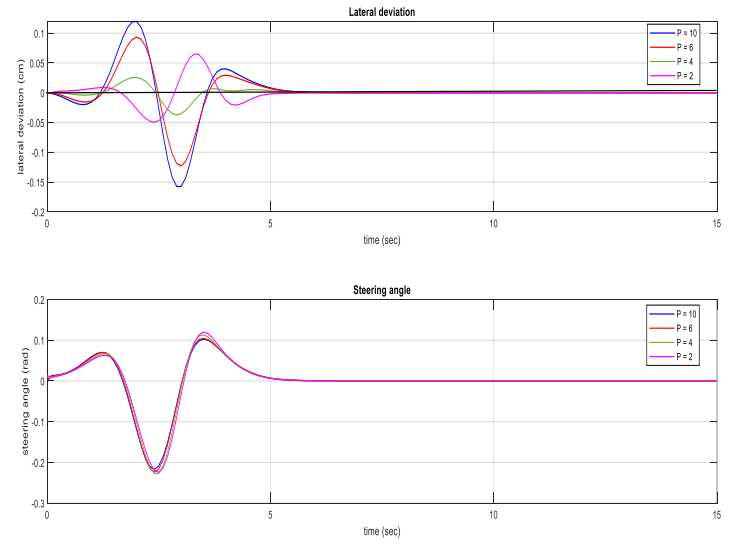

Fig 6. MPC response at different $\mathrm{P}$ time steps

From the figure, at the particular time step, Model predictive controller took care of the optimization issue over the prediction horizon fulfilling the constriants. The predicted pathway with the littlest $\mathbf{J}$ gives the ideal optimal solution and accordingly decides the ideal steering wheel angle that will get the vehicle as close as conceivable to the 
reference. At the current time step, MPC applies just the initial step of this ideal succession to the vehicle and ignores the rest. In view of the applied steering wheel angle, the will ventures some separation. At whenever step, the controller gets another estimation of the car's lateral position. It may be somewhat unique in relation to what the MPC controller has anticipated previously. This could be because of some unmeasured unsettling influence following up on the vehicle. For instance, it may be projected shadows or some other commotion. Presently the prediction horizon moves forward by one-time step and the controller rehashes similar pattern of computations to figure the ideal steering wheel angle for next time step as seen in the figure. It should be noted that optimal performance of the MPC was achieved at a lower longitudinal speed, even though the MPC controller was still able to achieve its goal of adjusting the steering angle toward the desired reference trajectory and a higher longitudinal speed also.
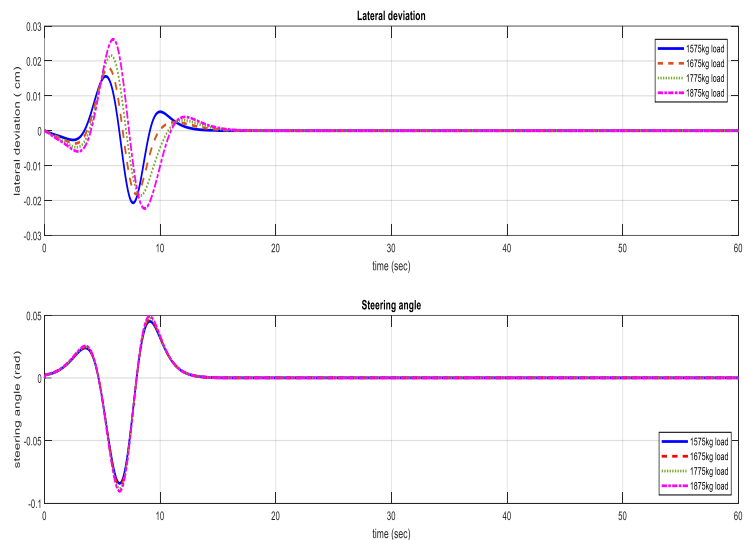

Fig 7. MPC Robustness Analysis at different values of load Change (mass)

From the figure, it can be seen that simulating at various conditions of mass didn't affect the MPC performance, although there was a little oscillation for the various masses, the optimizer was still able to solve the optimization problem and the controller tries to minimize the error between the reference and predicted path of the vehicle as seen in the figure. This was achieved because of the characteristics of the MPC in using the vehicle model to predict the future trajectory of the vehicle.

It also tries to minimize the change in the steering wheel angle from one time step to the next as seen in the simulation of the prediction horizon in figure 6 . The same thing can be seen in the simulation result at different front cornering stiffness in figure 4 . the different value of the front cornering stiffness did not affect much on the performance of the MPC, because of the ability of the MPC in predicting the vehicle future for the next $\mathrm{P}$ time steps as stated earlier.

Hence MPC showed a better performance and robustness because of its ability to use the vehicle model to predict the next future trajectory for the mobile robot to follow
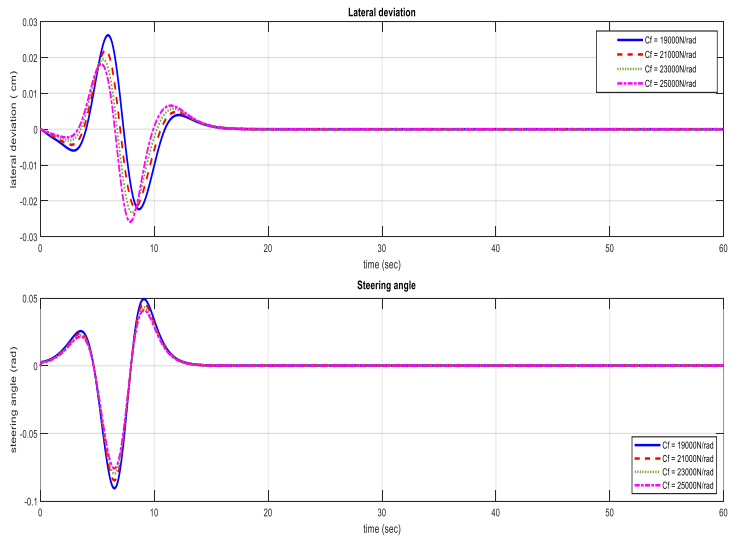

Fig 8. MPC Robustness Analysis at different values of Front Cornering Stiffness

\section{CONCLUSION}

The task of autonomously driving a car isn't an easy task. In an automated driving mode of the autonomous car, a control algorithm is used to control car's lateral movement. The objective of this algorithm is to keep the vehicle in the centre of its lane. Position and orientation of the vehicle can be determined by the use of sensors such as the camera used in identifying the white middle marker and left and right boundaries on the road and computer vision image processing technique used in [28].

\section{REFERENCES}

[1] M. Samuel, "A Review of some Pure-Pursuit based Path Tracking Techniques for Control of Autonomous Vehicle," vol. 135, no. 1, pp. 35-38, 2016.

[2] M. Syafiq and S. Mohamed, "Original Article Malaysian Drivers' Perception Towards Advanced Driver Assistance Systems ( ADAS )," vol. 2, no. 2, pp. 15-20, 2017.

[3] S. A. Adnan Shaout, Dominic Colella, "Advanced Driver Assistance Systems - Past, Present and Future.” 2011.

[4] H. Zhang and J. Wang, "Vehicle lateral dynamics control through afs/dyc and robust gain-scheduling approach," IEEE Transactions on Vehicular Technology, vol. 65, no. 1, pp. 489-494, 2016.

[5] G. Han, W. Fu, W. Wang, and Z. Wu, "The lateral tracking control for the intelligent vehicle based on adaptive PID neural network," Sensors, vol. 17, no. 6, 2017.

[6] J. Yang and N. Zheng, "An expert fuzzy controller for vehicle lateral control," in Proc. of IEEE Annual Conference on Industrial Electronics, 2007, pp. 880-885.

[7] X. Huang, H. Zhang, G. Zhang, and J. Wang, "Robust weighted gain scheduling $\mathrm{H} \infty$ vehicle lateral motion control with considerations of steering system backlash-type hysteresis," IEEE Transactions on Control Systems Technology, vol. 22, no. 5, pp. 1740-1753, 2014.

[8] C. Latrach, M. Kchaou, A. E. Hajjaji, and A. Rahbi, "Robust Hœ fuzzy networked control for vehicle lateral dynamics," in Proc. of IEEE Annual Conference on Intelligent Transportation Systems, 2013, pp. 905-910.

[9] R. Wang, Y. Sun, M. Lin, and H. Zhang, "Research on bus roll stability control based on LQR," in Proc. of International Conference on Intelligent Transportation, Big Data and Smart City, 2015, pp. $622-625$. 
[10] S. Lee and C. Chung, "Predictive control with sliding mode for autonomous driving vehicle lateral manoeuvring," in Proc. of American Control Conference, 2017, pp. 2998-3003.

[11] G. Tagne, R. Talj, and A. Charara, "Higher-order sliding mode control for lateral dynamics of autonomous vehicles, with experimental validation," in Proc. of IEEE Intelligent Vehicles Symposium, 2013, pp. 678-683.

[12] C. Hatipoglu, U. Ozguner, and K. Redmill, "Automated lane change controller design," IEEE Transactions on Intelligent Transportation Systems, vol. 4, no. 1, pp. 13-22, 2003.

[14] B. Gutjahr, L. Gr” oll, and M. Werling, "Lateral vehicle trajectory optimization using constrained linear time-varying MPC," IEEE Transactions on Intelligent Transportation Systems, vol. 18, no. 6, pp. 1586- 1595, 2017.

[15] J. Levinson, J. Askeland, J. Becker, J. Dolson, D. Held, S. Kammel, J. Kolter, D. Langer, O. Pink, V. Pratt, M. Sokolsky, G. Stanek, D. Stavens, A. Teichman, M. Werling, and S. Thrun, "Higher-order sliding mode control for lateral dynamics of autonomous vehicles, with experimental validation," in Proc. of IEEE Intelligent Vehicles Symposium, 2011, pp. 163-168.

[16] P. Falcone, F. Borrelli, J. Asgari, H. Tseng, and D. Hrovat, "Predictive active steering control for autonomous vehicle systems," IEEE Transactions on Control Systems Technology, vol. 15, no. 3, pp. 566-580, 2007.

[17]J.Ahmadi, A.Sedigh, and.Kabganian, “Adaptivevehiclelateral-plane motion control using optimal tire friction forces with saturation limits consideration," IEEE Transactions on Vehicular Technology, vol. 58, no. 8, pp. 4098-4107, 2009.

[18] J. Ni, J. Hu, and C. Xiang, "Envelope control for four-wheel independently actuated autonomous ground vehicle through AFS/DYC integrated control," IEEE Transactions on Vehicular Technology, vol. PP, no. 99, pp. 1-1, 2017.

[19] S.Dominguez,A.Ali, G.Garcia, and.Martinet, "Comparisonoflateral controllers for autonomous vehicle: Experimental Results," in Proc. of IEEE Conference on Intelligent Transportation Systems, 2016, pp. $1418-1423$.

[20] G. Tagne, R. Talj, and A. Charara, "Design and comparison of robust nonlinear controllers for the lateral dynamics of intelligent vehicles,"
IEEE Transactions on Intelligent Transportation Systems, vol. 17, no. 3, pp. 796-809, 2016.

[21] M. Samuel, M. Mohamad, M. Hussein, and S. Saad, "Development of Lane-keeping Controller Using Image processing," Int. J. Comput. Netw. Technol., vol. Volume 6, no. Issue 3, pp. 0-4, 2018.

[22]M. Samuel, M. Mohamad, S. M. Saad, and M. Hussein, "Development of Edge-Based Lane Detection Algorithm using Image Processing," JOIV Int. J. Informatics Vis., vol. 2, no. 1, pp. 19-22, 2018.

[23]M. A. Hassan and M. Samuel, "Study Of Vehicle Handling Characteristics In Design Of Autonomous Vehicle For Sustainable Transportation," vol. 4, no. 8, pp. 7857-7860, 2017.

[24] M. Samuel, "Comparative Analysis of Lane Detection Method Using Matlab,” no. December, pp. 25-31, 2016.

[25] A. Gray, Y. Gao, T. Lin, J. K. Hedrick, and F. Borrelli, "Stochastic predictive control for semi-autonomous vehicles with an uncertain driver model," in IEEE Conference on Intelligent Transportation Systems, Proceedings, ITSC, 2013, pp. 2329-2334, doi: 10.1109/ITSC.2013.6728575.

[26] H. M. Abdulsalam, O. T. Charles, and B. Ahmed, "The Influence of Silicon Carbide Particulate Loading on Tensile, Compressive and Impact Strengths of Al-Sicp Composite for Sustainable Development," vol. 63, no. Mmc, pp. 619-624, 2018, doi: 10.3303/CET1863104

[27] M. Samuel, "Performance and Robustness Analysis of Model Predictive Controller ( MPC ) and Proportional Integral Derivative Control ( PID ) of an Autonomous Vehicle for Lane Keeping Maneuvers," no. December, 2020, doi: 10.20944/preprints201912.0404.v1.

[28] M. Samuel, S. M. Ajibade, and F. F. Moveh, "A . I . Driven Thermal People Counting for Smart Window Facade Using Portable Low Cost Miniature Thermal Imaging S ensors," no. January, 2020, doi: 10.20944/preprints202001.0067.v1. 